reactive protein [CRP]), physician-diagnosed chronic conditions (diabetes, hypertension, stroke and Coronary heart disease) and depressive symptoms ascertained with the Center for Epidemiologic Studies Depression Scale range 0 to 8 . We controlled for age, sex and Apolipoprotein E (APOE).

Results Our findings suggest that experiencing financial hardship in childhood (20\% of the sample) was associated with a higher risk of cognitive impairment $(\mathrm{OR}=1.55,95 \%$ CI 1.15 - 2.09) in later life. A similar pattern was observed for having parents unemployed ( $7 \%$ of the sample) $(\mathrm{OR}=1.63,95 \% \mathrm{CI}$ $1.09-2.46)$ or physically abusive parents $(3 \%) \quad(\mathrm{OR}=3.21$, 95\% CI 1.82-5.66). We also found that increased depressive symptoms were interlinked with higher cognitive impairment, while APOEe4 and inflammatory markers were not directly associated. However, inflammation was indirectly associated with cognitive impairment, via depressive symptoms $(\beta=0.08$, $\mathrm{SE}=0.03, \quad \mathrm{p}=0.020)$ and chronic conditions $\quad(\beta=0.39$, $\mathrm{SE}=0.19, \mathrm{p}=0.042$ ).

Conclusion These findings support the psychosocial paradigm. They suggest that those from disadvantaged family backgrounds are more likely to have lower levels of education and be less wealthy, which in turn lead to poorer health, such as higher overall inflammation and increased depressive symptoms. These findings provide a plausible explanation for inequalities in late-life cognitive health.

\section{OP25 THE SEX-SPECIFIC ASSOCIATION BETWEEN MITOCHONDRIAL DNA HAPLOGROUPS AND TRAJECTORIES OF CARDIOMETABOLIC RISK FACTORS DURING CHILDHOOD AND ADOLESCENCE: A PROSPECTIVE COHORT STUDY}

${ }^{1} \mathrm{KN}$ O'Neill*, ${ }^{2} \mathrm{E}$ Aubrey, 'LM O'Keeffe. 'School of Public Health, University College Cork, Ireland; ${ }^{2}$ MRC Integrative Epidemiology Unit, University of Bristol, UK

\subsection{6/jech-2020-SSMabstracts. 25}

Background The sex-specific aetiology of cardiometabolic risk across the life course is well established but remains poorly understood. Mitochondria are essential for energy conversion in all cells. Mitochondrial DNA haplogroups have been implicated in the aetiology of cardiometabolic risk, though previous studies have not examined whether mitochondrial DNA haplogroups contribute to the sex-specific aetiology of cardiometabolic risk. We examined sex-specific associations between eight common European haplogroups and ten cardiometabolic risk factors in childhood and adolescence.

Methods Longitudinal data from the Avon Longitudinal Parents and Child Study, a prospective birth cohort study, was analysed. Cardiometabolic risk factors included systolic blood pressure (SBP), diastolic blood pressure (DBP), pulse rate, triglycerides, high-density lipoprotein cholesterol (HDL-c), nonHDL-c, insulin, glucose, height-adjusted fat mass and heightadjusted lean mass, measured at research clinics when participants were approximately 7, 9, 10, 11, 13, 15 and 18 years old. Participants were genotyped using the SNP genotyping platform by Sample Logistics and Genotyping Facilities at the Wellcome Trust Sanger Institute and LabCorp (Laboratory Corporation of America). We used linear spline multilevel models to examine the association between mitochondrial DNA haplogroups and cardiometabolic risk factor trajectories across childhood and into adolescence. Analysis was stratified by sex to allow for differential effects between sexes. All trajectories were modelled in MLwiN version 2.36 called from Stata version 15 .

Results Sample sizes for different outcomes range from 2,023 females and 1,962 males (6,518 total measures) for insulin up to 3,570 females and 3,689 males (17,039 total measures) for triglycerides. There was no strong evidence that haplogroups H,J,K,U,T and I are associated with cardiometabolic risk factors. In females, haplogroup $\mathrm{X}$ was associated with $3.22 \mathrm{mmHg}$ (95\% confidence interval (CI):0.46.0) lower SBP at age 7 and $1.29 \mathrm{~kg}$ (95\%CI:0.4-2.2) lower lean mass at age 9 compared with haplogroup $H$. Similar associations were observed at age 18 , albeit with CIs spanning the null. Haplogroup $\mathrm{X}$ was also associated with $15.5 \%$ (95\% CI:2.5-28.5) lower fat mass at age 9 in females, although this association did not persist at age 18 . Males with haplogroup W had higher HDL-c and lower non-HDL-c at birth, with decreasing and increasing rates of change, respectively, during the first 9 years of life. The associations did not persist at age 18 .

Conclusion Mitochondrial DNA haplogroups X and W may play a role in the sex-specific aetiology of cardiometabolic risk during childhood and adolescence.

\section{OP26 ADVERSE CHILDHOOD EXPERIENCES, POPULATION ATTRIBUTABLE RISK AND INCREMENTAL RISK OF CORONARY HEART DISEASE: A 13 YEARS FOLLOW-UP OF THE WHITEHALL II COHORT STUDY}

${ }^{1}$ M Akasaki*, ${ }^{2} \mathrm{D}$ Batty, ${ }^{3} \mathrm{~A}$ Steptoe, ${ }^{4} \mathrm{O}$ Nicholas, ${ }^{2} \mathrm{C}$ Valencia-Hernández, ${ }^{1} \mathrm{R}$ Hardy, ${ }^{3} \mathrm{~J}$ Abell. ${ }^{1}$ Department of Social Science, University College London, London, UK; ${ }^{2}$ Department of Epidemiology and Public Health, University College London, London, UK; ${ }^{3}$ Department of Behavioural Science and Health, University College London, London, UK; ${ }^{4}$ Department of Statistical Science, University College London, London, UK

\subsection{6/jech-2020-SSMabstracts. 26}

Background Adverse childhood experiences (ACEs), such as parental divorce, parental mental illness, parental separation, have been increasingly recognised as an upstream potential causal factor of development and premature mortality of coronary heart disease (CHD). No studies, however, have investigated attributable risk for incident $\mathrm{CHD}$ by each and all types of ACEs, and associations between combinations of ACEs and incident CHD.

Methods Among 5149 participants aged 35 to 55 at entry (1985-1988) to the Whitehall II cohort study with follow-up of 12.9 years, we examined; (i) associations between ACEs and incident CHD; (ii) what extent removal of each and all ACEs could eliminate incident CHD; and (iii) incremental risk of CHD in the association with ACEs combinations. Cox proportional hazard regression was applied to estimate hazard ratios and 95\% confidence intervals. After identifying a model, we computed the average marginal effects, and hazard ratios and 95\% confidence intervals for each ACEs combination. Incident CHD was identified through the Hospital Episode Statistics (HES) from Phase 5 (1997-1999) when ACEs were measured, to Phase 11 (2012-2013).

Results In the study sample, $65.6 \%$ had at least one ACE. After adjusting for sex, age, ethnicity, and childhood socioeconomic status, none of ACEs ('parental attachment', 'financial hardship', 'parental punishment', 'parental dysfunction', 'early-life parental separation', 'orphanage', 'hospitalisation') had statistically significant associations with incident CHD. All types of ACEs were attributed to approximately $13 \%$ of 
incident $\mathrm{CHD}$, on average $1.9 \%$ by each ACE. Estimated hazards of CHD are more likely to depend on ACEs combinations than counts of ACEs. For instance, those who experienced three ACEs (financial hardships, early-life parental separation, and hospitalisation) had 1.52 times higher hazard (95\%CI: 1.10 to 2.12), while those who had four ACEs (poor parental attachment, financial hardships, parental harsh punishment, and early-life parental separation) had 1.31 times higher hazard (95\%CI: 1.03 to 1.67$)$, than those did not have ACEs.

Conclusion The findings of this study show that there is incremental risk of CHD in ACEs combinations. As the ACEs are more likely to co-occur, even if an individual ACE had no associations with increased risk of $\mathrm{CHD}$, it is crucial to intervene in ACEs holistically. By taking away all types of ACEs, we estimated that $13 \%$ probability of developing CHD could be eliminated. This finding can be scientific evidence for early childhood framework for intervention to reduce health inequalities over life course, which are originated in early life.

\section{Thursday 10 September}

\section{Non-Communicable Disease: Multi-Morbidity}

\section{OP27 SOCIODEMOGRAPHIC AND LIFESTYLE PREDICTORS OF INCIDENT HOSPITAL ADMISSIONS WITH MULTIMORBIDITY IN A GENERAL POPULATION 1999- 2019: THE EPIC-NORFOLK COHORT}

${ }^{1}$ RN Luben*, 'SA Hayat, ${ }^{2}$ NJ Wareham, ${ }^{1}$ PD Pharoah, ${ }^{1} \mathrm{KT}$ Khaw. ${ }^{1}$ Department of Public Health and Primary Care, University of Cambridge School of Clinical Medicine, Cambridge, UK; ${ }^{2}$ MRC Epidemiology Unit, University of Cambridge School of Clinical Medicine, Cambridge, UK

\subsection{6/jech-2020-SSMabstracts.27}

Background The ageing population and prevalence of longterm disorders with multimorbidity is a major health challenge worldwide. Patients with multimorbidity account for a disproportionately high share of healthcare workload and costs and experience reduced wellbeing and quality of life. The associations between comorbid conditions and mortality risk are well established; however, few prospective community-based studies have reported on prior risk factors for incident hospitalisations with multimorbidity. Our study examines the independent associations for a range demographic, lifestyle and physiological determinants and the likelihood of subsequent hospital incident multimorbidity using a prospective community-based cohort of middle-aged and older men and women resident in Norfolk, UK. We explore demographic, lifestyle and physiological exposures including age and sex, body mass index, cigarette smoking, alcohol intake, educational attainment, occupational social class, physical activity, plasma vitamin C, total cholesterol, systolic blood pressure and common prevalent diseases.

Methods Incident hospital admissions with multimorbidity were examined in 25014 men and women aged 40-79 in EPIC-Norfolk, a British prospective population-based study initially recruited in 1993-1997 and followed-up until 2019. The determinants of incident multimorbidity, defined as Charlson Comorbidity Index $\geq 2$, were examined in multivariable models for the 10-year period 1999-2009 and repeated with independent measurements in a second 10 -year period 2009-2019.

Results Between 1999-2009 18179 participants (73\% of the population) had a hospital admission. Baseline 5-year and 10 year incident multimorbidity were observed in $11 \%$ and $21 \%$ of participants respectively. Age per 10-year increase OR 2.13 (95\% CI 2.03-2.23) and male sex OR 1.28 (95\% CI 1.17-1.39) predicted incident multimorbidity over 10 years. In the subset free of the most serious diseases at baseline, current smoking OR 1.71 (95\% CI 1.53-1.91), BMI >30 $\mathrm{kg} / \mathrm{m}^{2}$ OR 1.37 (95\% CI 1.24-1.51) and physical inactivity OR 1.09 (95\% CI 1.00-1.18) were positively associated and plasma vitamin $\mathrm{C}$ (a biomarker of plant food intake) per SD increase OR 0.88 (95\% CI 0.84-0.91) inversely associated with incident 10-year multimorbidity after multivariable adjustment for age, sex, social class, education, alcohol consumption, systolic blood pressure and cholesterol. Results were similar when re-examined for a further time period 2009-2019.

Conclusion Age, male sex and potentially modifiable lifestyle behaviours including smoking, physical inactivity and low fruit and vegetable intake were associated with increased risk of future incident hospital admissions with multimorbidity.

\section{OP28 MULTIMORBIDITY AND FIT NOTE RECEIPT IN WORKING AGE ADULTS WITH LONG-TERM HEALTH CONDITIONS}

\begin{abstract}
1,2SK Dorrington*, 'E Carr, ${ }^{1,3}$ SAM Stevelink, ${ }^{1} \mathrm{C}$ Woodhead, ${ }^{1} \mathrm{~J}$ Das-Munshi, ${ }^{4} \mathrm{M}$ Ashworth, ${ }^{2} \mathrm{M}$ Broadbent, ${ }^{5} \mathrm{I}$ Madan, 'S Hatch, ${ }^{1,2} \mathrm{M}$ Hotopf. ${ }^{1}$ Psychological Medicine, Institute of Psychiatry, Psychology and Neuroscience King's College London, London, UK; ${ }^{2} \mathrm{NIHR}$ Biomedical Research Nucleus, South London and Maudsley NHS Trust, London, UK; ${ }^{3}$ King's Centre for Military Health Research, Institute of Psychiatry, Psychology and Neuroscience, London, UK; ${ }^{4}$ School of Population Health and Environmental Sciences, King's College London, London, UK; ${ }^{5}$ Department of Occupational Health, Guy's and St Thomas NHS Foundation Trust, London, UK
\end{abstract}

10.1136/jech-2020-SSMabstracts.28

Background Research on sickness absence has typically focused on single diagnoses, despite increasing recognition that longterm health conditions are highly multimorbid and clusters comprising coexisting mental and physical conditions are associated with poorer clinical and functional outcomes. The digitisation of sickness certification in the UK offers an opportunity to address sickness absence in a large primary care population.

Methods Lambeth Datanet is a primary care database which collects individual level data on GP consultations, prescriptions, Quality and Outcomes Framework (QOF) diagnostic data, sickness certification (fit note receipt) and demographic information (including age, gender, self-identified ethnicity, and truncated postcode). We analysed 326,415 people's records covering a 40-month period from January 2014 to April 2017.

Results We found significant variation in multimorbidity by demographic variables, most notably by self-defined ethnicity. Multimorbid health conditions were associated with increased fit note receipt. Comorbid depression had the largest impact on first fit note receipt, more than any other comorbid diagnoses. Highest rates of first fit note receipt after adjustment for demographics were for comorbid epilepsy and depression (HR 4.0; 95\% CI 3.4-4.6), followed by chronic pain and depression (HR 3.9; 95\% CI 3.5-4.4) and cardiac condition and depression (HR 3.9; 95\% CI 3.2-4.7). 